\title{
Quantum theory of preparation and measurement
}

\author{
DAVID T. PEGG \\ School of Science, Griffith University, Nathan, Brisbane, \\ Q 4111, Australia \\ STEPHEN M. BARNETT and JOHN JEFFERS \\ Department of Physics and Applied Physics, University \\ of Strathclyde, Glasgow G4 0NG, Scotland
}

PACS numbers: 03.65.Ta, 03.67.Hk

Author for correspondence: D. T. Pegg

Phone: +61 73875 7152,Fax: +613875 7656, EDallkgg@sct.gu.edu.au 


\begin{abstract}
The conventional postulate for the probabilistic interpretation of quantum mechanics is asymmetric in preparation and measurement, making retrodictidiant on inference by use of Bayes' theorem. Here we present a more fundamentaktsixem postulate from which both predictive and retrodictive probabilities emerge imatedi even where measurement devices more general than those usually considemed a involved. We show that the new postulate is perfectly consistent with the conventional postulate.
\end{abstract}




\section{Introduction}

The conventional formalism of quantum mechanics based on the Copenhagen interpretation is essentially predictive. We assign a state to a soed on our knowledge of a preparation event and use this state to predict the probabilities of outcomes of future measurements that might be made on the system. If we have sufficient knowledge to assign a pure state, then this state contains thmumaxamount of information that nature allows us for prediction. With less knowledge, we can only assign a mixed state. This formalism works successfully. Sometimes, howemay have knowledge of the result of a measurement and wish to retrodict the statedprepa A particular example of this is in quantum communication where the recipienteseae quantum system that the sender has prepared and sent. If the prepared state has not evolved at the time of measurement to an eigenstate of the operator represtmeti recipient's measurement, then the best retrodiction that the recipient care nisato calculate probabilities that various states were prepared. While it isblposoi do this by using the usual predictive formalism and inference based on Bayes' theoremhi\$1]s $t$ often quite complicated. Aharonet al. [2], in investigating the origin of the arrow of time, formulated a retrodictive formalism that involves assigning a sasted bn knowledge of the measurement outcome. This state is assigned to the system ju\$o prior the measurement and evolves backward in time to the preparation event. While this formalism seems to offer a more direct means of retrodiction, Belinfante s[3rghtmd that the formalism is only valid in very particular circumstances teattially involve the prepared states, which in his case are eigenstates of a preparationtonpdraving a 
flat a priori probability distribution. While the lack of preparation knowledge associated with such an unbiased distribution is sometimes applicable, in general it is not.

In our recent work [4 - 6] we have found quantum retrodiction useful for a variety of applications in quantum optics. Furthermore the formalism can be generadised to be applicable when there is not a dlatriori probability distribution for the prepared states by using Bayes' theorem [6]. The price of this generalisatioarsappebe a loss in symmetry between preparation and measurement. In this paper we adopt $\mathrm{d}$ forma approach to investigate this question more closely. We find that we can replaacalthe us measurement postulate of the probability interpretation of quantum mechanics by a fundamental postulate that is symmetric in measurement and preparation. Thiss alls to formulate a more general theory of preparation and measurement than that of the conventional formalism and makes clear the relationship between the predianide retrodictive approaches. The new postulate also allows us to see clearhfamels argument in an appropriate perspective. We show that our new postulate is ๓ntirely $\mathrm{i}$ accord with the conventional postulate. The retrodictive formalism results samtae calculated experimental outcomes of quantum mechanics as does the conventional approach despite the fact that we ascribe a different state to the slystwmen preparation and measurement.

\section{Preparation and measurement devices}

We consider a situation where Alice operates a device that prepares a quantum system and Bob does subsequent measurements on the system and records the results. The preparation device has a readout mechanism that indicates the state then siss 
prepared in. We associate a preparation readout eventhere $i=1,2, \cdots$, of the

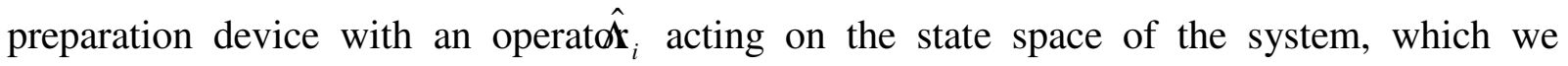
call a preparation device operator (PDO). Thisratpe not only represents the prepared state but also contains information about any bimasts preparation. A bias might arise, for example, because the device may not be abprodduce certain states or Alice may choose rarely to prepare other states. We descthee operation of the preparation device mathematically by a set of PDOs. The measurementicae also has a readout mechanism that shows the result of the measurement. We associ measurement readout event, where $j=1,2, \cdots$, of the measurement device with a measurement cdewiperator (MDO) $\hat{\Gamma}_{j}$ acting on the state space of the system. Thistoperepresents the state of the system associated with the measurement and contains infation about any bias on the part of Bob or the device in having the measurement recbrdFor example for a von Neumann measurement the MDO would be proportional to a psudee projector. We describe the operation of the measurement device mathematicaby a set of MDOs. In general the operators $\hat{\Lambda}_{i}$ need not be orthogonal to each other, and nomedoperators $\hat{\Gamma}_{j}$.

In order to eliminate the complication of time ution we assume for now that the system does not change between preparation medsurement. For example, there may not be a sufficiently long time between præparaand measurement for evolution to occur. In an experiment Alice chooses a staprepare and, when the readout mechanism indicates that this state has been susfady prepared, the preparation readout event $i$ is automatically sent to a computer for recordigigb then measures the system. If he chooses, he may then send the nerasut readout evenj obtained to the computer for recording. If the computer receivesecord from both Alice and Bob it 
registers combined event $(i, j)$. The measurement device may not produce a readout event corresponding to every possible preparationert and different preparation events may lead to the same measurement readout eventereTts not necessarily a uniform probability that Bob will record all readout eventWhe preparation device may be capable of preparing only a limited number of sstatahere is not necessarily a uniform probability that Alice will choose to prepare hadket states. The experiment is repeated many times with Alice choosing states to prepareshes wishes and Bob recording the measurement readout events he chooses. The computroduces a list of combined events $(,, j)$ from to each experiment, from which various aeare frequencies can be found.

We may wish tpredict the measurement result that will be recorded in a particular experiment on the basis of our knowledget the actual preparation eveintnd our knowledge of the operation of the measuringiadevthat is, of the set of MDOs. Because of the nature of quantum mechanics, we lussuaannot do this with certainty, the best we can do is to calculate the probabilithiat various possible states will be detected and recorded by Bob. Similarly the bestcan do inetrodicting the preparation event recorded by Alice in a particudxperiment on the basis of our knowledge of the recorded measurement evginand our knowledge of the set of PDOs for the preparation device, is to calculate probitiei for possible preparation events. Our aim in this paper is to postulate a fundameratationship that allows us to calculate such predictive and retrodictive probabilities, whi could then be compared with the occurrence frequencies obtained from the collectiof combined event $(s i, j)$ recorded 
by the computer. In this way a theory of quartnodiction is verifiable experimentally.

Difficulties have arisen in studying retrodiction] [because the usual formulation of quantum mechanics is predictive. That is, measent theory is formulated in terms of predicting measurement outcomes. In order topkpreparation and measurement as well as prediction and retrodiction on a symmeftoreting, it is convenient to reformulate the probability interpretation of quantum mechanibsy means of postulate (1) below. We show that this leads to the conventional asymimqtredictive postulate and, as an assurance that our approach is perfectly equivalent predictive theory, in the Appendix we derive postulate (1) from conventional measuremeheory.

\section{Fundamental postulate}

A sample space of mutually exclusive outcomes canconstructed from the collection of recorded combined events by identingyithese events with points of the space so that identical events are identified thith same point. A probability measure assigns probabilities between zero and one to thintp such that these probabilities sum to unity for the whole space. The probability assigto a poin(ti,j) is proportional to the number of combined ever(tis $j$ ) identified with that point, that is, to the camerr frequency of the even(i,j). Our fundamentalostulate in this paper for the probabilistic interpretation of quantum mechanics is that thebpability associated with a particular point $(i, j)$ in this sample space is

$$
P^{\Lambda \Gamma}(i, j)=\frac{\operatorname{Tr}\left(\hat{\Lambda}_{i} \hat{\Gamma}_{j}\right)}{\operatorname{Tr}(\hat{\Lambda} \hat{\Gamma})}
$$


where the trace is over the state space of thensysand

$$
\begin{aligned}
& \hat{\Lambda}=\sum_{i} \hat{\Lambda}_{i} \\
& \hat{\Gamma}=\sum_{j} \hat{\Gamma}_{j} .
\end{aligned}
$$

In order to ensure that no probabilities are negative assume tha $\hat{\AA}_{i}$ and $\hat{\Gamma}_{j}$ are nonnegative definite. If a combined event from arerixpnt chosen at random is recorded then expression (1) is the probability for thatnete be $i,(j)$. That is, expression (1) is the probability that the state prepared by Alicresponds to $\hat{\Lambda}_{i}$ and the state detected by Bob corresponds $t \hat{\Phi}_{j}$, given that Bob has recorded the associated meansent event. The essence of the postulate lies in therator of (1); the denominator simply ensures that the total probability for all the rreed mutually exclusive outcomes is unity. We note that the fundamental expression orill)y requires $\hat{\Lambda}_{i}$ and $\hat{\Gamma}_{j}$ to be

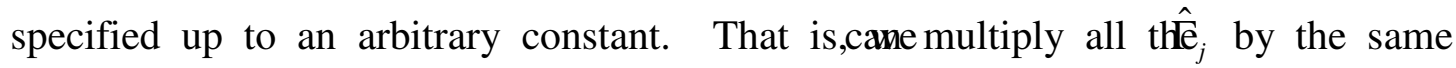
constant without affecting $P^{\Lambda \Gamma}(i, j)$ and similarly foo $\hat{\mathbf{A}}_{i}$. We use this flexibility later to choose $\hat{\Gamma}_{j}$ for convenience such th $\hat{\mathrm{k}}-\hat{\Gamma}$ is non-negative definite, wheile is the unit operator. We shall also use this flexibility ioscing $\hat{\Lambda}_{i}$. From (1) we can deduce the following probabilities: 


$$
\begin{aligned}
& P^{\Lambda \Gamma}(i)=\sum_{j} P^{\Lambda \Gamma}(i, j)=\frac{\operatorname{Tr}\left(\hat{\Lambda}_{i} \hat{\Gamma}\right)}{\operatorname{Tr}(\hat{\Lambda} \hat{\Gamma})} \\
& P^{\Lambda \Gamma}(j)=\frac{\operatorname{Tr}\left(\hat{\Lambda} \hat{\Gamma}_{j}\right)}{\operatorname{Tr}(\hat{\Lambda} \hat{\Gamma})} \\
& P^{\Lambda \Gamma}(j \mid i)=\frac{P^{\Lambda \Gamma}(i, j)}{P^{\Lambda \Gamma}(i)}=\frac{\operatorname{Tr}\left(\hat{\Lambda}_{i} \hat{\Gamma}_{j}\right)}{\operatorname{Tr}\left(\hat{\Lambda}_{i} \hat{\Gamma}\right)} \\
& P^{\Lambda \Gamma}(i \mid j)=\frac{\operatorname{Tr}\left(\hat{\Lambda} \hat{\Gamma}_{j}\right)}{\operatorname{Tr}\left(\hat{\Lambda} \hat{\Gamma}_{j}\right)}
\end{aligned}
$$

Expression (4) is the probability that, if an expment chosen at random has a recorded combined event, this event includes preparation that the recorded combined event includes the meanent eventj. Expression (6) is the probability that, if the recorded combined eventludes eventi, it also includes evgint That is, it is the probability that the event dedoby Bob is the detection of the state corresponding to $\Gamma_{j}$ if the state prepared by Alice in the experinoentsponds to $\hat{\Lambda}_{i}$. Expression (6) can be obtained by limiting theplarspace to those events containing and is essentially Bayes' formula [7]. Likewise i\$7the probability that the state prepared by Alice corresponds $\hat{\mathbf{s}}_{i}$ if the event recorded by Bob is the detectidme of $\mathrm{t}$ state corresponding $\mathrm{to}_{j}$.

Expression (6) can be used for prediction. Inrourdecalculate the required probability from our knowledge of the PDî associated with the preparation event 
we must also know every possible MÎ́ $\oint$ that is, we must know the mathematical description of the operation of the measuring devisimilarly we can use (7) for retrodiction if we $\operatorname{know} \hat{\boldsymbol{E}}_{j}$ and all the $\hat{\mathbb{A}}_{i}$ of the preparation device.

\section{Unbiased devices}

\subsection{A priori probability}

Of all the states that Alice might prepare, therend priori probability, which is independent of the subsequent measurement, that shleooses a particular one. For $P^{\Lambda \Gamma}(i)$ in (4) to represent thaspriori probability the expression $\mathrm{f}^{\Lambda \Gamma}(i)$ must be independent of the operation of measurement deviceA specific condition must be imposed on the measuring device and its operationda this. This condition is that the set of MDOs describing the operation of the meanen device must be such that their sum $\hat{\Gamma}$ is proportional to the identity operator on space of the system, that is

$$
\hat{\Gamma}=\gamma \hat{1}
$$

say where $\gamma$ is a positive number. Then we can repplaue the numerator and

denominator in (4) by the unit operator and thememfe of $\hat{\Gamma}$ is removed from the expression, making $P^{\Lambda \Gamma}(i)$ equal to $P^{\Lambda}(i)$ where the latter is defined as

$$
P^{\Lambda}(i)=\frac{\operatorname{Tr} \hat{\Lambda}_{i}}{\operatorname{Tr} \hat{\Lambda}}
$$


Expression (9) is the $\mathbb{E}_{j}$-independent, $a$ priori probability that the state prepared by Alice corresponds to $\hat{\Lambda}_{i}$.

It is useful also to define an operator

$$
\hat{\rho}_{i}=\frac{\hat{\Lambda}_{i}}{\operatorname{Tr} \hat{\Lambda}_{i}}
$$

The trace of $\hat{\rho}_{i}$ is unity so these non-negative operators dexesity operators describing the states Alice may prepare. From the definiti(0)s and (10) we can write as proportional to $P^{\Lambda}(i) \hat{\rho}_{i}$. The constant of proportionality always cancelshe expressions for the various probabilities so thereno loss of generality in taking this constant to be unity. Then we have

$$
\hat{\Lambda}_{i}=P^{\Lambda}(i) \hat{\rho}_{i}
$$

We see explicitly from (11) how the Pĥे as well as representing the prepared state, also contains information about the bias in itppation. The biasing factor is simply the $a$ prioripreparation probability.

From (9), (11) and (2) we see thath has unit trace so it also is a density operator given by

$$
\hat{\Lambda}=\hat{\rho}=\sum_{i} P^{\Lambda}(i) \hat{\rho}_{i}
$$


This is the best description we can give of the stapared by Alice if we do not know which particular preparation or measurement evenolt place but we do know the possible states she can prepare and therioriprobabilities associated with each.

\subsection{Unbiased measurements}

We call the operation of a measurement device froichw(8) is true, and thus $P^{\Lambda \Gamma}(i)=P^{\Lambda}(i)$, unbiased. Not all measurements are unbiased, as we shodusd later, but for now we shall focus on measuring devicek unibiased operations. For these it is convenient to define

$$
\hat{\Pi}_{j}=\frac{\hat{\Gamma}_{j}}{\gamma} \quad .
$$

From (6), (8) and (10) we then obtain

$$
P^{\Lambda \Gamma}(j \mid i)=\operatorname{Tr}\left(\hat{\rho}_{i} \hat{\Pi}_{j}\right)
$$

From (13) and (8) the sum $\hat{\Gamma} f f$ is the unit operator, so these non-negative operators form the elements ofpณobability operator measure(POM) [8]. Our result (14) is thfundamental postulate of quantum detection theor8]. Thus our postulate (1) reduces to the conventional postulate for unbiasadasurements. Expressions (14) and 
(10) allow us to identify the PDí for the preparation of a pure state as being proportional to the corresponding pure state prøgerc

It is worth remarking on the asymmetry of (14hatnthe PDO has become a density operator and the MDO has become a POM eltemen the simple case where both the PDO and the MDO are pure state projectarsfor a von Neumann measurement of a pure state, symmetry is restored. In genbraw,ever, density operators and POM elements have quite different normalisation propiest. The asymmetry in preparation and measurement, and hence a time asymmetry, doots arise here through some basic asymmetry in quantum mechanics. Rather it arisomfour request that the probability for Alice's choice of preparation event be indertdof subsequent measurement. This is usually an implicit assumption in the conventlonthat is predictive, probability interpretation of quantum mechanics. The apparastymmetry is reinforced by adopting (14) as a fundamental postulate of measurement theas done for example by Helstrom [8].

A simple, but important, example of unbiased meanent is the case where no measurement is made. For example the measuringicaemight not interact with the system at all and thus gives a meter reading of foer all prepared states. As there is only one measurement readout event, there is ondg $\operatorname{MDO} \hat{\Gamma}_{j}=\hat{\Gamma}$. The only probability that we can assign to a preparationntevif we do not know the preparation readout event and if we have made no measurementhonsystem is the priori probability $P^{\Lambda}(i)$. Thus if we calculate the retrodictive probabifite $(i \mid j)$ on the basis of the no-measurement state, then we must obtiin $(i)$. From (7) and $(\hat{\mathcal{T}})$, must therefore be proportional to the unit operator sadthe measurement must be unbiased. 
The single POM element for the measuring device tnmes to ensure that the sum of the elements is the unit operator.

The operation of most ideal measuring devices isallus unbiased, but this is not always the case. In [6] we discussed two-photorfënence for photons from a parametric down-converter where results from higheumber states are discarded. Another example is in the operational phase meaments of Noht al. [9]. Here certain photo-detector readings are not recorded becauseythdo not lead to meaningful values of the operators being measured. The probabilitised for the experimental statistics are then suitably renormalised.

\subsection{Unbiased preparation}

We say in general that the operation of a preparalevice is unbiased if the PDOs $\hat{\Lambda}_{i}$ are proportional t $\hat{\overline{\boldsymbol{\sigma}}}_{i}$ where

$$
\sum_{i} \hat{\Xi}_{i}=\hat{1}
$$

that is, if the operato $\hat{\overline{\mathrm{S}}}_{i}$ form the elements of a preparation device POMen, Tfbr a preparation device with an unbiased operatio $\mathbb{R}^{\Lambda \Gamma}(j)$ is independent of $\hat{\mathbf{A}}_{i}$ and

$$
P^{\Lambda \Gamma}(i \mid j)=\operatorname{Tr}\left(\hat{\Xi}_{i} \hat{\rho}_{j}^{\text {retr }}\right)
$$

where 


$$
\hat{\rho}_{j}^{\text {retr }}=\hat{\Gamma}_{j} / \operatorname{Tr} \hat{\Gamma}_{j}
$$

A specific example of a preparation device withunbiased operation is where Alice prepares a spin-half particle in the up orndstate, each with a probability of onehalf. The two preparation device operatôrs and $\hat{\Lambda}_{\text {down }}$ can then be taken as proportional to density operators given by the expe projectors $|u p\rangle\langle u p|$ and $\mid$ down $\backslash$ down|. Then $\hat{\Lambda}$ is proportional to the unit operator on the sqpatee of the particle and we find from (7) that

$$
P^{\Lambda \Gamma}(u p \mid j)=\operatorname{Tr}\left(|u p\rangle\langle p| \hat{\rho}_{j}^{\mathrm{retr}}\right)
$$

which gives the retrodictive probability that thes in which Alice prepared the particle was the up state if Bob detected the $\hat{s p p t r t e ~}^{\text {retr }}=\hat{\Gamma}_{j} / \operatorname{Tr} \hat{\Gamma}_{j}$. This is consistent with (16) with $\hat{\Xi}_{u p}=|u p X u p|$.

Many preparation devices have biased operations, (sb6) is not applicable to them. For example the preparation of a fieldphoten number state may be constrained through limited available energy. His tcase the set of PDOs would not include projectors for higher photon number states thus could not sum to be proportional to the unit operator in the wholee s\$ptace of the field. Alternatively, Alice might prepare the spin-half particle in the upe stat in an equal superposition of the up 
and down states only. For such situation we mustthes more general form of the retrodictive probability (7).

\section{Time evolution}

In the conventional approach, when the state ofenyschanges unitarily between preparation and measurement, we replac, $\hat{\boldsymbol{\rho}}_{i}$ by $\hat{\rho}_{i}\left(t_{m}\right)=\hat{U} \hat{\rho}_{i} \hat{U}^{\dagger}$ in the appropriate probability formulae where $\hat{U}$ is the time evolution operator between the prepiorat time $t_{p}$ and the measurement timte. Thus in this paper we replấceby $\hat{\Lambda}_{i}\left(t_{m}\right)=\hat{U} \hat{\Lambda}_{i} \hat{U}^{\dagger}$ while noting that $\left.\operatorname{T} \hat{\boldsymbol{U}} \hat{\Lambda}_{i} \hat{U}^{\dagger}\right)=\operatorname{Tr} \hat{\Lambda}_{i}$. This is clearly consistent with (10) and yields the usual predictive formula (14) $\hat{p}_{t}$ threplaced by $\hat{\rho}_{i}\left(t_{m}\right)$. For the retrodictive probability replacing (7) we obtainsing the definition (17),

$$
P^{\Lambda \Gamma}(i \mid j)=\frac{\operatorname{Tr}\left(\hat{U} \hat{\Lambda}_{i} \hat{U}^{\dagger} \hat{\rho}_{j}^{\text {retr }}\right)}{\operatorname{Tr}\left(\hat{U} \hat{\Lambda} \hat{U}^{\dagger} \hat{\rho}_{j}^{\text {retr }}\right)}
$$

From the cyclic property of the trace we can reethrits as

$$
P^{\Lambda \Gamma}(i \mid j)=\frac{\operatorname{Tr}\left[\hat{\Lambda}_{i} \hat{\rho}_{j}^{\text {retr }}\left(t_{p}\right)\right]}{\operatorname{Tr}\left[\hat{\Lambda} \hat{\rho}_{j}^{\text {retr }}\left(t_{p}\right)\right]}
$$

where $\hat{\rho}_{j}^{\text {retr }}\left(t_{p}\right)=\hat{U}^{\dagger} \hat{\rho}_{j}^{\text {retr }} \hat{U}$ is the retrodictive density operator evolved backdsa in time to the preparation timeThis is the retrodictive formula we obtained prasiy [6] using the conventional approach and Bayes' theorem [N.e note that (20) can be interpreted 
as the state collapse taking place at the preparatime $t_{p}$. This arbitrariness in when we choose to say the collapse occurs is not confinedetrodiction. Even the conventional predictive formula obtained from (14) by replacip $\left.\hat{p}_{i} t_{m}\right)$ by $\hat{\rho}_{i}\left(t_{m}\right)=\hat{U} \hat{\rho}_{i} \hat{U}^{\dagger}$ can be rewritten as $\operatorname{Tr}\left(\hat{\rho}_{i} \hat{U}^{\dagger} \hat{\Pi}_{j} \hat{U}\right)$ where $\hat{U}^{\dagger} \hat{\Pi}_{j} \hat{U}$ can be interpreted as an element of a POM describing the operation of a different measuringide for which the measurement event takes place immediately after the preparation time

\section{Example}

As an important example of our approach, we apply this section to the experimental situation envisaged by Belinfante [3]After studying the work of Aharonov et al[2], Belinfante came to the conclusion that retrodiction only valid in very special circumstances. He examined the situation where easurement device B makes von Neumann measurements with outcomes corresponding to complete set of pure states $\left|b_{j}\right\rangle$. His preparation device, which prepares pureesst|at $\left.t_{i}\right\rangle$, comprises a measuring device A making von Neumann measurements on a systa a state given by a density operator $\hat{\rho}_{g}$. The predictive probability that the state mealsuis $\left|b_{j}\right\rangle$ if the state prepared is $\left|a_{i}\right\rangle$ is $\left.\left\langle a_{i} \mid b_{j}\right\rangle\right|^{2}$. Belinfante argued that quantum theory wouldirbe- $\mathrm{t}$ symmetric in its probability rules if the retrodietprobability that the state prepared is $\left|a_{i}\right\rangle$, if the state measured $\left|b_{j}\right\rangle$, is taken a $\left|\left\langle b_{j} \mid a_{i}\right\rangle\right|^{2}$, which is the retrodictive inverse of $\left.\left\langle a_{i} \mid b_{j}\right\rangle\right|^{2}$. These two expressions are equal. Belinfanteleded that retrodiction is 
valid only if the mixed state of the system befiensurement by A is uniformly "garbled", that is if the density operafogris proportional to the unit operator.

Let us examine this situation in terms of our flosma The operation of the von Neumann measuring device B is unbiased so we canribe it by a set of PDOs which form a POM with elements

$$
\hat{\Gamma}_{j}=\hat{\Pi}_{j}^{b}=\left|b_{j} X_{b_{j}}\right|
$$

Similarly the operation of the measuring devices Adescribed by the POM with elements $\hat{\Pi}_{i}^{a}=\left|a_{i}\right\rangle\left\langle a_{i}\right|$. Thea priori probability for state $\hat{\rho}_{i}=\left|a_{i}\right\rangle\left\langle a_{i}\right|$ to be prepared $\operatorname{ikr}\left(\hat{\rho}_{g} \hat{\Pi}_{i}^{a}\right)$. Thus from (11) we have

$$
\hat{\Lambda}_{i}=\operatorname{Tr}\left(\hat{\rho}_{g}\left|a_{i} X a_{i}\right|\right)\left|a_{i} X a_{i}\right|
$$

From (14), the predictive probability for an undalameasuring device, we find that the probability that the state measured $\left|b_{j}\right\rangle$ if the state prepared $\left|i_{s_{j}}\right\rangle$ is $\left.\left\langle a_{i} \mid b_{j}\right\rangle\right|^{2}$. This agrees with Belinfante's result. However, the additative probability (7) becomes, from (21) and (22)

$$
P^{\Lambda \Gamma}(i \mid j)=\frac{\left.\operatorname{Tr}\left(\hat{\rho}_{g}\left|a_{i}\right\rangle\left\langle a_{i}\right|\right)\left\langle a_{i} \mid b_{j}\right\rangle\right|^{2}}{\sum_{i}\left[\left.\operatorname{Tr}\left(\hat{\rho}_{g}\left|a_{i}\right\rangle\left\langle a_{i}\right|\right)\left\langle a_{i} \mid b_{j}\right\rangle\right|^{2}\right]}
$$


for the probability that the state preparedaij if the state measured $\left|z_{j}\right\rangle$. This agrees with the result of Belinfante if, and only $\hat{\rho}_{g}$ ifs proportional to the unit operator.

From the above, we see that the difficulty witdodietion raised by Belinfante is due to use of the retrodictive inverse of an inppionte predictive formula. Belinfante effectively found $P^{\Lambda \Gamma}(i \mid j)$ by taking the retrodictive inverse $\operatorname{Bf}^{\Gamma}(j \mid i)$ in (14). However (14) is valid only for unbiased measuriagicas and its retrodictive inverse, which is given by (16), is only valid for unbiarequation devices. It is not surprising then that Belinfante found his retrodictive formudaly worked $i \hat{p}_{g}$ is proportional to the unit operator as this is precisely the conditioeded to ensure that the PDOs (22) describe the operation of an unbiased preparatiøvide. For biased preparation we must use the retrodictive inverse of theore general predictive formula (6) which is just (7) as used above. We conclude that retrodiction is vfidid a general preparation device provided the correct formula is used.

\section{Conclusion}

Overall, the approach adopted in this paper toptbbability interpretation of quantum mechanics puts preparation and measurememt a more equal footing than in the conventional approach where preparation is ulsyaignored and the measuring device is assumed to be unbiased. We have formulated pproach in terms of more general sets of non-negative definite operators than POMWe have found that for an unbiased measuring device, for which the measuring devicerapors reduce to the elements of a POM, the preparation device operators can be wmittas density operators, absorbing the normalisation denominator in the general expressiq6). This reduces (6) to (14), the 
conventional asymmetric postulate of quantum detieat theory. Just as (14) is only applicable for unbiased measuring devices, its oetictive inverse (16) is only applicable for unbiased preparation devices. These latterides are unusual in practice, which leads to Belinfante's objection to retrodiction. usteful theory of retrodiction requires that allowance be made for bias in the preparadionice. A fully symmetric probability interpretation of quantum mechanics would then alsequire allowance to be made for a biased measurement device as we have done in thpierp

As mentioned in the introduction, the retrodictfoemalism results in the same calculated experimental outcomes of quantum mechasias does the conventional approach based on the Copenhagen interpretation,sfice the fact that we ascribe a different state to the system between preparationd measurement. In the conventional approach, the state assigned to the system contains information needed to predict the outcomes of possible measurements on the system. this sense, the conventional approach is essentially predictive in nature andthiss a legitimate part of the broader picture that also includes retrodiction. Indeed donventional approach is sufficient in the sense that one can perform retrodictive probiabicalculations by using it together with Bayes' theorem. On the other hand, this apprös not necessary in that one could perform predictive probability calculations, albeitomplicated, using the retrodictive formalism plus Bayes' theorem. Thus both the conimaal and retrodictive formalisms should be viewed merely as means for calculatingbapilities with one being more convenient than the other depending on the situmtioWe should also mention, however, that retrodiction also raises interesting philosaphl questions if one wishes to ascribe a physical existence or reality to the state in theløgical sense. These issues go beyond 
trying to decide if the state of the system iłly"rethe predictive or the retrodictive state. In [5] it is shown that it is possibdlee foetrodictive state to be entangled for some situations where there is no entanglementhin predictive picture. In the predictive formalism, the Many-Worlds interpretation [10] depsi an increasing number of branching universes that include the different pibse results of measurements as we go forward in time. In the retrodictive formalismanyAWorlds interpretation should look very different. Presumably the branching will øcas we go backwards in time from the measurement to the preparation. We do not intendursue such questions here. As long as the retrodictive formalism yields the actrquantum mechanical probabilities, we view it as an acceptable and sometimes more eamerst approach to quantum mechanics and shall leave the philosophical isstesmetaphysics.

\section{Acknowledgments}

This work was supported by the Australian Researchuncil and the U.K. Engineering and Physical sciences Research Council. SMB thathks Royal Society of Edinburgh and the Scottish Office Education and Lifelong hiage Department for the award of a Support Research Fellowship.

\section{Appendix}

In this appendix we derive our general postulate f $f(x \mathrm{~m}$ the standard predictive postulate (14). As we have already shown how f(110)ws from (1), this establishes 
that (1) is both necessary and sufficient for cbepted probability interpretation of quantum mechanics.

The operation of the measuring deviakused by Bob is described by the set of MDOs $\hat{\Gamma}_{j}$ with $j=1,2, \cdots$. As discussed earlier, we choose for convenieneearbitrary constant $\operatorname{in} \hat{\Gamma}_{j}$ such that $\hat{\boldsymbol{C}}$ is non-negative definite. This allows us to edefiset of non-negative definite operators $\hat{\Pi}_{k}$ by

$$
\begin{aligned}
& \hat{\Pi}_{j}=\hat{\Gamma}_{j} \quad \text { for } j=1,2, \cdots \\
& \hat{\Pi}_{0}=\hat{1}-\hat{\Gamma} .
\end{aligned}
$$

It is clear from (3) that the operatîn ${ }_{h}$ sum to the unit operator and thus form the elements of a POM. We can use this POM to defiropetration of another measuring device $\bar{M}$ which has precisely the same operation as that, o£xcept that it allows an extra measurement eventk $=0$ to be recorded. The readout for this event can be interpreted as "none of the evenits We can use the usual postulate corresponding $t$ (14) to obtain the probability that measurementneke will be recorded b $\overline{M I}$ if the system is prepared in stat, $\hat{\boldsymbol{\rho}}_{i}$ as

$$
P^{\Lambda \Pi}(k \mid i)=\operatorname{Tr}\left(\hat{\rho}_{i} \hat{\Pi}_{k}\right)
$$

Thus 


$$
P^{\Lambda \Pi}(i, k)=\operatorname{Tr}\left(\hat{\rho}_{i} \hat{\Pi}_{k}\right) P^{\Lambda}(i)
$$

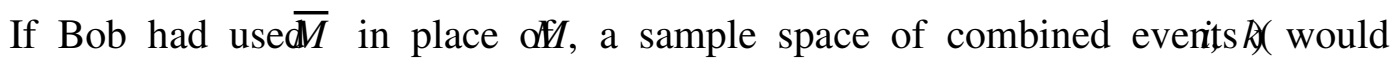
have been obtained that is larger than that of teven obtained with $M$ in that it includes some extra pointsi, (0). If these extra events are ignored, then flhereahice between the operations $\overline{\mathrm{o}} \overline{\mathbb{G}}$ and $M$ vanishes, so the restricted sample space of events $(i, k)$ with $k \neq 0$ will be the same as the sample space of evientsfor $M$. The probability $P^{\Lambda \Gamma}(i, j)$ will thus be equal to the probability of findiagetlent $i(A)$, with $k$ not zero, in this restricted sample space. Thibapility will be equal $\mathrm{t} B^{\Lambda \Pi}(i, j)$ with a normalisation factor to ensure that the total pbibitay for the restricted sample space is unity. From (A4), (A1) and from the definitionw(3)then have

$$
\begin{aligned}
P^{\Lambda \Gamma}(i, j)= & \frac{\operatorname{Tr}\left(\hat{\rho}_{i} \hat{\Gamma}_{j}\right) P^{\Lambda}(i)}{\sum_{i, j} \operatorname{Tr}\left(\hat{\rho}_{i} \hat{\Gamma}_{j}\right) P^{\Lambda}(i)} \\
& =\frac{\operatorname{Tr}\left(\hat{\rho}_{i} \hat{\Gamma}_{j}\right) P^{\Lambda}(i)}{\operatorname{Tr}(\hat{\rho} \hat{\Gamma})}
\end{aligned}
$$

where $\hat{\rho}$ is defined by (12). If we now intrôtudey defining it as being proportional to $P^{\Lambda}(i) \hat{\rho}_{i}$, which is consistent with (10), and defineby (2), we find that (A 5) reduces to 


$$
P^{\Lambda \Gamma}(i, j)=\frac{\operatorname{Tr}\left(\hat{\Lambda}_{i} \hat{\Gamma}_{j}\right)}{\operatorname{Tr}(\hat{\Lambda} \hat{\Gamma})}
$$

in agreement with (1).

\section{References}

[1] Box, G. E. P. and Tiao, G. C., 187zßesian inference in statistical analysis (Sydney: Addison-Wesley), p. 10

[2] Aharonov, Y., Bergman, P. G. and Lebowitz,.,J.1964,Phys. Rev.134, B1410;

Penfield, R. H., 1966łm. J. Phys34, 422; Aharonov, Y. and Albert, D. Z., 1984 Phys. Rev. D29, 223; Aharonov Y. and Albert, D. Z., 1984;s. Rev. D29, 228; Aharonov, Y. and Vaidman, L., 199.1,Phys. A: Math. Ge24, 2315.

[3] Belinfante, F. J. 1975Measurements and Time Reversal in Objective Quantum Theory (Oxford: Pergamon Press).

[4] Barnett, S. M. and Pegg, D. T., 1996)ys. Rev. Lett 76, 4148; Pegg, D. T., Barnett, S. M. and Phillips, L. S., 199.7,mod. Optic,s44, 2135; Pegg, D. T., Phillips, L. S. and Barnett, S.M. 1998,hys. Rev. Lett81,1604; Phillips, L, Barnett, S. M. and. Pegg, D. T., 1998hys. Rev. A 58, 3259; Pegg D. T. and Barnett, S. M., 1999, Quantum and Semiclass. Op1, 442; Barnett, S. M. and Pegg, D. T., 1999, Phys. Rev.A, 60, 4965; Barnett, S. M., Pegg, D. T., Jeffers, dJ.Jedrkiewicz, O., 2000,J. Phys. B: At. Mol. Opt. P.h33, 3047; Barnett, S. M., Pegg, D. T., Jeffers, J., Jedrkiewicz, O. and Loudon, R., 2010yys. Rev. A62, 022313;

Barnett, S. M., Pegg, D. T., Jeffers, J. and Jendikxं, O., 2001Phys. Rev. Lett., 86, 2455. 
[5] Pregnell, K. L. and Pegg, D. T., 2001mod. Optic48, 1293.

[6] Barnett, S. M., Pegg D. T. and Jeffers, JQ, 200nod. Optic,\$47, 1779.

[7] Boas, M. L. 198Mathematical Methods in the Physical Sciend(New York: Wiley), p. 695.

[8] Helstrom, C. W., 197Quantum Detection and Estimation Theor@New York: Academic Press)

[9] Noh, J. W., Fougerés and Mandel, L., 199Bys, Rev. Lett.16, 2579.

[10] Everett, H., 1957Rev. Mod. Phy\& 29, 454. 